\title{
Research and Implementation of Constant Current Source Charger Han Guanglin ${ }^{1, a}$, Zeng $X u^{1, b}$, Zhang Yanling ${ }^{1, c}$
}

\author{
${ }^{1}$ College of Electronic and Communication Engineering, Tianjin Normal University, Tianjin 300387 , \\ China \\ ahgltj@163.com, benghnly@foxmail.com, c1850042994@qq.com
}

Keywords: Rectifier; Constant Current Power Supply; Full Automatic Power-off

\begin{abstract}
: this paper introduces a kind of the rectifier circuit, adjustable constant current charging circuit, detecting voltage circuit, display circuit and power circuit of six parts of constant current source charger design scheme. The charger using op-amp composition adjustable constant current charge, constant current source circuit to avoid the constant voltage charging and lack of such as output voltage will vary with load, using a 555 timer consists of oscillator and general TL082 J FET dual operational amplifier, compared with $3 \mathrm{~V}$ voltage reference test voltage circuit to realize full automatic disconnection functions.
\end{abstract}

\section{Introduction}

Nowadays, for the battery has been the focus of people care about, right and good charging method can ensure the battery life.So some constant voltage and constant current charge appeared, while constant voltage charging can satisfy normal charging, but the output voltage will change due to load changes.And The vast majority of constant current circuit is regulated by using the three-step chip to stabilize voltage charging.In this paper, the charger can not only at a constant current for two batteries recharged but also output voltage does not change with load. It can automatically displayed when the battery is full of charge, and stopped charging automatically cut off power supply.

\section{Design of this project ${ }^{1}$}

The overall block diagram simple constant-current battery charger 5 as shown in figure 1 is made up of rectifier circuit, adjustable constant current circuit, detecting voltage circuit, display circuit and charging power supplying circuit five parts to form a complete charging circuit.

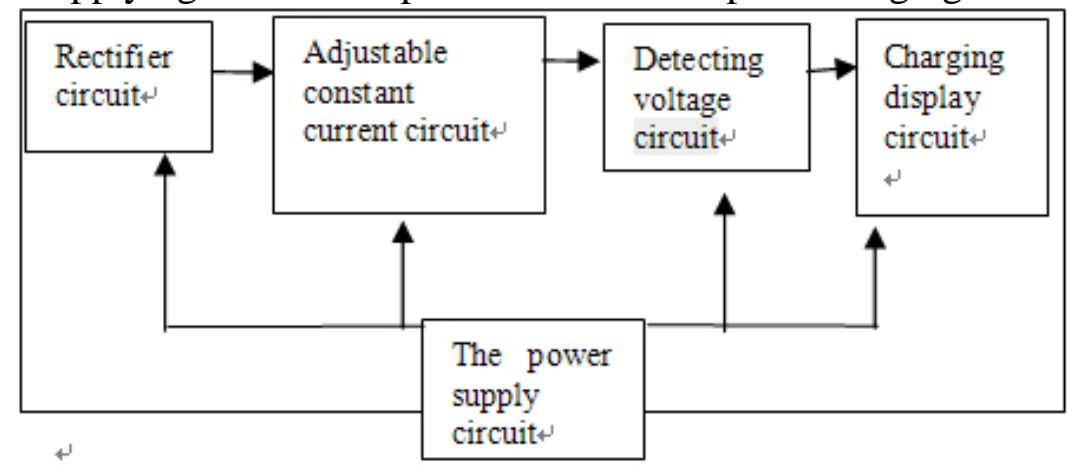

Fig.1. simple battery overall block diagram of adjustable constant current charging circuit

The function of the transformer rectifier circuit is $220 \mathrm{~V}$ alternating current (AC) in the grid will be converted to the appropriate current and voltage signals, thus providing signal for subsequent circuit.The function of adjustable constant current circuit is charging constantly current source circuit by using adjustable constant current consisted of op-amp composition.The function of detecting voltage circuit is the device of comparing, compare the voltage across the battery control relay work, so as to realize automatically cut off power supply when a full charge.The function of the circuit of charging display are using 555 thanks oscillator with LED will stop charging status and status display of the charging circuit.The function of the regulated power supply circuit is 
providing all of the above circuit DC voltage.

\section{The main unit circuit design}

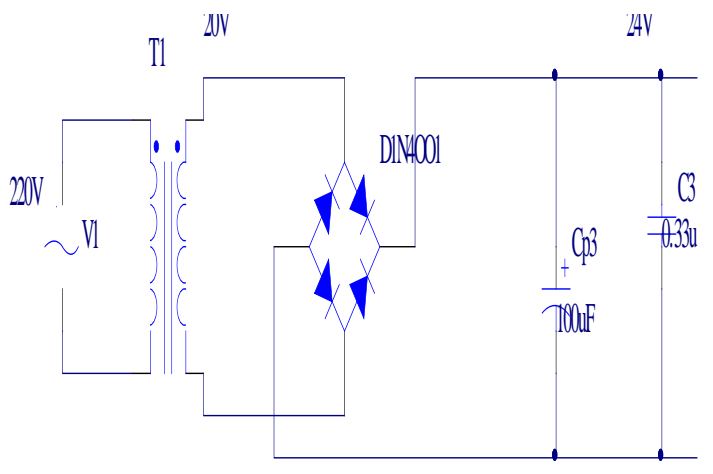

Fig.2. rectifier circuit $^{3}$

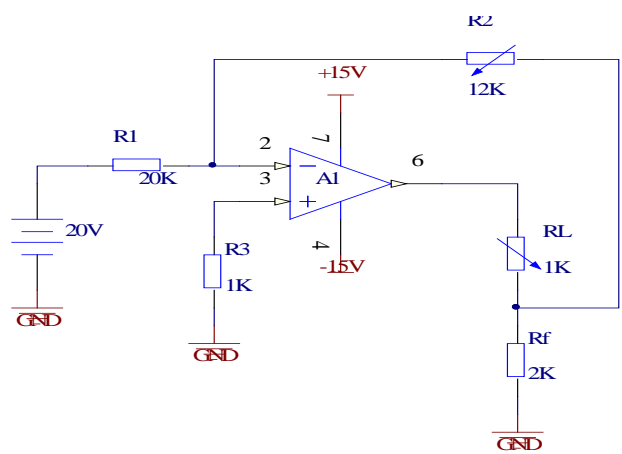

Fig.3. adjustable constant current circuit $^{2}$

As shown in figure 2 is transformer rectifier circuit, which consists of transformer, diode bridge type circuit, capacitance.The transformer USES conventional core transformer, and the grid of 220 $\mathrm{V}$ AC to $20 \mathrm{~V} \mathrm{AC}$, and then through the diode bridge type circuit rectifier and capacitor Cp3 and C3 filtering.As to in order to get a smooth load voltage tau $\mathrm{d}=\mathrm{RL}(3 \sim 5)$ or more $\mathrm{T} / 2$, $\mathrm{T}$ for the cycle of the power supply voltage, the rectifier circuit resistance is not too big and discharge time constant for on relations, $\mathrm{V}=(1.1 \sim 1.2) \mathrm{V} 2$.

As shown in figure 3 is adjustable constant current source circuit, composed of operational amplifier.R2 in the circuit is greater than the Rf, due to the inverting input is empty, and the amplifier input current is very small, can think all flow to the current through the R1, R2 and there Es/R1 = IL Rf / + R2 (Rf), so as shown in figure each element values changed to let IL of the R2 value is greater than $4 \mathrm{~mA}$, the greater the R2, the greater the IL.And IL has nothing to do with the amplifier parameters for the constant current source, and adjust the size of the constant current will adjust with the R2 changed, satisfying the requirement of different types of battery charging. When changes in RL, IL can stable, because it is a current negative feedback circuit in parallel.Through the simulation result shows that the circuit of current adjustable range is $4 \mathrm{~mA} 40.63 \mathrm{~mA}$, corresponding to the $\mathrm{R} 2$ value range is $12 \mathrm{k}$ to $160 \mathrm{k} .(\mathrm{R} 1, \mathrm{R} 2, \mathrm{RL}, \mathrm{Rf}$ visual value)

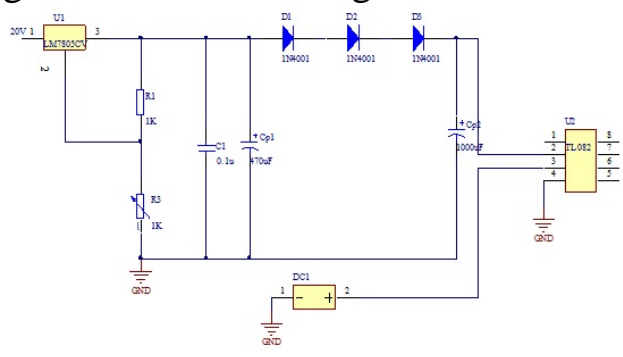

Fig.4. test voltage circuit

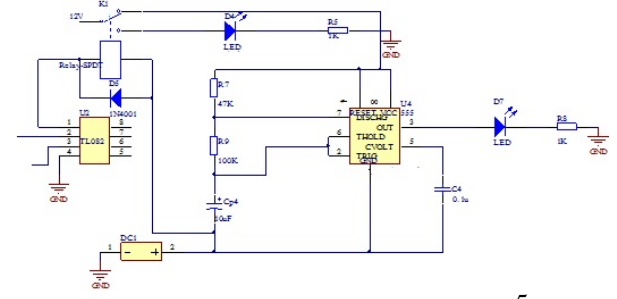

Fig.5. charging display circuit ${ }^{5}$

From figure 4,LM7805 is stationary three-terminal voltage regulator integrated circuit, its nominal output voltage of $+5 \mathrm{~V}$, resistor voltage divider $\mathrm{R} 1$ and $\mathrm{R} 3$ consist of the device of sharing the voltage, three-terminal voltage regulator integrated block partial pressure points in 7805 to end GND2 feet, adjust the device of sharing the voltage of R3 potential change integrated land end, so it can change the size of the output voltage of the circuit. The maximum output current $1.5 \mathrm{~A}$.When the device of sharing the voltage slide to the top, equivalent to 2 feet ground to end, the output voltage is equal to the nominal output voltage of $5 \mathrm{~V}$ of manifold blocks. Sliding points down, the output voltage increases, the maximum can make the output reached $10 \mathrm{~V}$, output voltage and the resistance of the relationship between the partial pressure can be expressed as: the output $=\mathrm{V}(1+$ R3 / R1) : in the nominal output voltage of $7805 \mathrm{~V}, 5 \mathrm{~V}$, when the largest R3, output $=5 *(1+$ $1000 / 1000)=10$ V.LM7805 output terminal 3 feet by the diode D1 to D3 positive pressure drop tube after processing can be regulated voltage $3 \mathrm{~V} \sim 8 \mathrm{~V}$, through capacitance contact international like applying filter to send out, as a reference voltage.By adjusting the reference voltage to batteries to 
charge more, when at the ends of the battery voltage is lower than the reference voltage, TL082 output is low level, and when the battery reaches $3 \mathrm{~V}$, output is high level, so as to control the switch of the relay.

From figure 5 The frequency of the part of display is produced by 555 time-based circuit is 0.4 $\mathrm{Hz}$, duty ratio is $1 / 5$ of the square wave signal, drive LED light.This circuit uses the NE555 integrated block of oscillator. Its circuit includes two processes: one is charging Cp4 by using of DC power through the R7 and R9, the second is the resistance R9 discharge process. Through these two alternating operation of the process,then output electric terminal $\mathrm{Q}$ can produce the pulse signal in the NE555 integrated circuit.

When charging, relay doesn't work, 1 and 2 of switch K1 normally closed, 555 chip began to work, when the battery voltage increase gradually, since 555 to termination of the positive pole of the battery, lead to the rise of potential, LED flicker dark gradually.

TL082 when filled with high output voltage, the relay began to work, normally closed off, normally open point closed, 555 control flashing diode extinguishes, 1 and 3 of the K1 closed, D4 lighted, indicating the battery.

NE555 supply of the output current is big,promoting a variety of automatic control of load directly.

\section{Conclusion}

The main characteristics of the charger circuit output voltage is not changing with load constant current charging, and can achieve the function of automatic power.It is by the rectifier circuit, the adjustable constant current circuit, detection circuit of voltage, charging display circuit four parts.Has the charging current is adjustable, full of power function. The charger is not only to overcome the influence of load on the output voltage and meet the needs of different users, also ensure the service life of the battery.

\section{Acknowledgment}

In this paper,I learned how to use drawing software such as Orcad16.3 summer 09 and simulation software, had a more profound understanding of DC regulated power supply, strengthened the module and electrical knowledge, had the further understanding of the principle of the circuit, the function of each components, features a more familiar understanding, in the process of design, of course, we also encountered many difficulties, when making the power charger, on how to implement a full electric power automatically after a piece of it is lost, then look for the related literature in the library electronic resources,search some similar circuit design of material on the Internet,and improved ours by absorbing its essence. This process make the ability of combining theory and practice of experiment has improved further.All in all, I have benefited a lot from this design.

\section{References}

[1]Hua chengying, Tong ShiBai, analog electronic technology foundation (fourth edition), higher education press, may, 2006

[2]Any boat, electronic system design, zhejiang university press, June 2001

[3]Fu-an yao, electronic circuit design and practice of shandong science and technology press, October 2001

[4]Wang, circuit and digital logic design practice, southeast university press, October 1999

[5]Li Yinhua, electronic circuit design instruction, Beijing university of aeronautics and astronautics press, June 2005 